\title{
Overtone Modes in an At-Cut Quartz Trapped-Energy Resonator with Elliptical Electrodes
}

\author{
LONG LI AND ZHI WANG* \\ School of Mechanics and Engineering Science, Zhengzhou University, Zhengzhou 450001, China
}

(Received October 28, 2016; in final form January 6, 2017)

\begin{abstract}
We analyze thickness-shear vibrations of an at-cut quartz plate with elliptical electrodes. The electrodes are essentially optimal in the sense of Mindlin in that they approximately satisfy the criterion for the Bechmann number in every direction. The scalar differential equation derived by Tiersten and Smythe is used which allows us to obtain the overtone modes in addition to the fundamental modes.
\end{abstract}

DOI: 10.12693/APhysPolA.131.294

PACS/topics: quartz, resonator, thickness-shear vibration, elliptical, overtone modes

\section{Introduction}

Electrode shape, size, and thickness are important design parameters. Early studies on electrode geometry were scattered. An important result was given by Mindlin [1] who obtained the optimal electrode shape and size for at-cut quartz resonators. The optimal electrodes in [1] satisfy the criterion for the Bechmann number in every direction so that when the resonator is in thickness-shear (TSh) vibration the entire electroded region vibrates in phase without charge cancellation on the electrodes. Due to the in-plane anisotropy of quartz plates, the Mindlin optimal electrodes are nearly elliptical, with the major axis exceeding the minor axis by $25 \%$. Subsequent studies on elliptical electrodes can be found in [2-7]. For at-cut quartz plates, the optimal electrodes [1, 4], the frequencies and mode shapes in plates with elliptical electrodes [5] were all determined using the Mindlin firstorder plate theory and thus were limited to the fundamental TSh modes with one nodal point along the plate thickness only. In this paper we extend these results to overtone TSh modes using the scalar differential equations by Tiersten and Smythe [8] which are valid for both the fundamental and the overtone TSh modes.

\section{Governing equations}

Consider an unbounded plate of at-cut quartz as shown in Fig. 1. The electrode stiffness is neglected. The electrode boundary is given by

$$
\frac{x_{1}^{2}}{a^{2} M_{n} / c_{55}}+\frac{x_{3}^{2}}{a^{2}}=1 .
$$

The semi-major and semi-minor axes are $a \sqrt{M_{n} / c_{55}}$ and $a$, respectively. $M_{n}$ and $c_{55}$ are to be defined later. The ratio between the major and minor axes is about 1.26 which is close to the optimal electrodes determined in [1]. We analyze the unelectroded and electroded regions separately.

\footnotetext{
*corresponding author; e-mail: wangzhi@zzu.edu.cn
}

The scalar equations governing the electroded and unelectroded plates are, respectively, given by [8]:

$$
\begin{aligned}
& M_{n} \frac{\partial^{2} u_{1}^{n}}{\partial x_{1}^{2}}+c_{55} \frac{\partial^{2} u_{1}^{n}}{\partial x_{3}^{2}}+\rho\left(\omega^{2}-\bar{\omega}_{n}^{2}\right) u_{1}^{n}=0, \\
& M_{n} \frac{\partial^{2} u_{1}^{n}}{\partial x_{1}^{2}}+c_{55} \frac{\partial^{2} u_{1}^{n}}{\partial x_{3}^{2}}+\rho\left(\omega^{2}-\omega_{n}^{2}\right) u_{1}^{n}=0,
\end{aligned}
$$

where

$$
\begin{aligned}
& M_{n}=c_{11}+\left(c_{12}+c_{66}\right) r+\frac{4\left(r \bar{c}_{66}-c_{66}\right)\left(r c_{22}+c_{12}\right)}{c_{22} n \pi \kappa} \\
& \quad \times \cot \frac{\kappa n \pi}{2} \bar{c}_{66}=c_{66}+\frac{e_{26}^{2}}{\varepsilon_{22}}, \\
& \kappa=\left(\frac{\bar{c}_{66}}{c_{22}}\right)^{1 / 2}, \quad r=\frac{c_{12}+c_{66}}{\bar{c}_{66}-c_{22}}, \quad R=\frac{2 \rho^{\prime} h^{\prime}}{\rho h}, \\
& \bar{\omega}_{n}^{2}=\frac{n^{2} \pi^{2} \hat{c}_{66}}{4 h^{2} \rho}<\omega_{n}^{2}=\frac{n^{2} \pi^{2} \hat{c}_{66}}{4 h^{2} \rho}, \\
& \hat{c}_{66}=\bar{c}_{66}\left(1-\frac{8 \bar{k}_{26}^{2}}{n^{2} \pi^{2}}-2 R\right), \quad \bar{k}_{26}^{2}=\frac{e_{26}^{2}}{\bar{c}_{66} \varepsilon_{22}} .
\end{aligned}
$$

\section{Theoretical analysis}

We introduce a new coordinate system $\left(\xi_{1}, \xi_{3}\right)$ by

$$
x_{1}=\xi \sqrt{M_{n} / c_{55}}, x_{3}=\xi_{3} .
$$

In this coordinate system, the elliptical electrodes in Eq. (1) are represented by a circular domain described by

$$
\frac{\zeta_{1}^{2}}{a^{2}}+\frac{\zeta_{3}^{2}}{a^{2}}=1 .
$$

Equations (2) and (3) become

$$
\begin{aligned}
& c_{55} \nabla^{2} u_{1}^{n}+\rho\left(\omega^{2}-\bar{\omega}_{n}^{2}\right) u_{1}^{n}=0, \\
& c_{55} \nabla^{2} u_{1}^{n}+\rho\left(\omega^{2}-\omega_{n}^{2}\right) u_{1}^{n}=0 .
\end{aligned}
$$

We then introduce a polar coordinate system $(r, \theta)$ :

$$
\xi_{3}=r \cos \theta, \xi_{1}=r \sin \theta .
$$

We are only interested in modes that are independent of $\theta$. Then Eqs. (7) and (8) reduce to

$$
\frac{\partial^{2} u_{1}^{n}}{\partial r^{2}}+\frac{1}{r} \frac{\partial u_{1}^{n}}{\partial r}+\alpha^{2} u_{1}^{n}=0, \quad r<a,
$$




$$
\frac{\partial^{2} u_{1}^{n}}{\partial r^{2}}+\frac{1}{r} \frac{\partial u_{1}^{n}}{\partial r}-\beta^{2} u_{1}^{n}=0, \quad r>a,
$$

where

$$
\begin{aligned}
& \alpha^{2}=\rho\left(\omega^{2}-\bar{\omega}_{n}^{2}\right) / c_{55}>0, \\
& \beta^{2}=\rho\left(\omega_{n}^{2}-\omega^{2}\right) / c_{55}>0 .
\end{aligned}
$$

The general solutions to Eqs. (10) and (11) are

$$
u_{1}^{n}=\left\{\begin{array}{l}
A J_{0}(\alpha r), r<a, \\
B K_{0}(\beta r), r>a,
\end{array}\right.
$$

where $J_{0}$ is the zero-order Bessel function of the first kind which is bounded at the origin. $K_{0}$ is the zero-order modified Bessel function of the second kind which decays exponentially at infinity. At the electrode edge we have the continuity of $u_{1}^{n}$ and its radial derivative

$$
\begin{aligned}
& A J_{0}(\alpha a)=B K_{0}(\beta a), \\
& A J_{0}^{\prime}(\alpha a) \alpha=B K_{0}^{\prime}(\beta a) \beta,
\end{aligned}
$$

where a prime represents differentiation with respect to the entire argument, i.e., $\alpha r$ or $\beta r$. For nontrivial solutions of $A$ and/or $B$, the determinant of the coefficient matrix must vanish, which gives the following frequency equation that determines $\omega$ :

$$
\alpha J_{0}^{\prime}(\alpha a) K_{0}(\beta a)-\beta J_{0}(\alpha a) K_{0}^{\prime}(\beta a)=0 .
$$

\section{Numerical results and discussion}

Consider an at-cut quartz plate with $2 h=1 \mathrm{~mm}$ and $R=0.01[4,5]$. We begin with the same fundamental mode of the same resonator as that in $[4,5]$ with $a=9 \mathrm{~mm}$. Only one trapped mode is found with $\omega=10347460.121 / \mathrm{s}$ which is very close to the $\omega=103400741 / \mathrm{s}$ in $[4,5]$. This shows the agreement between the Mindlin plate equations and the scalar equations in [8]. The corresponding mode shape is shown in Fig. 2.

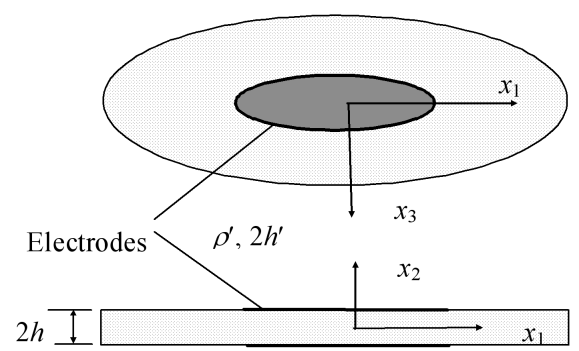

Fig. 1. A quartz plate with elliptical electrodes.
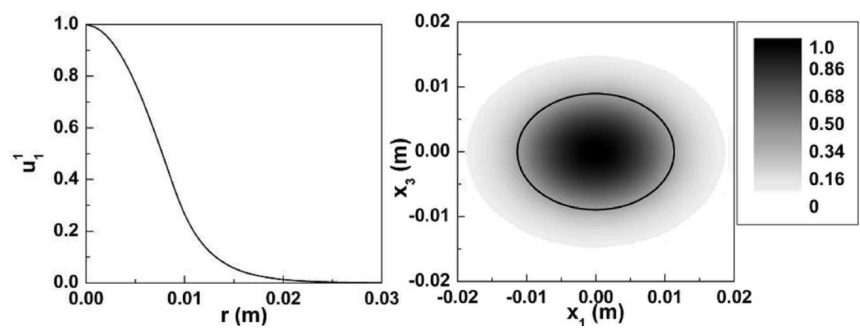

Fig. 2. Trapped mode when $n=1, R=0.01$, and $a=9 \mathrm{~mm}$.
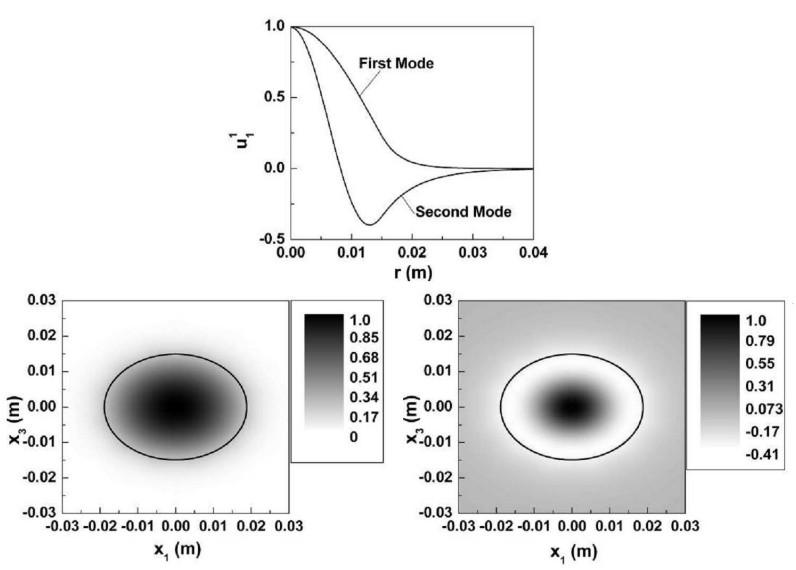

Fig. 3. Trapped modes when $n=1, R=0.01$, and $a=15 \mathrm{~mm}$.
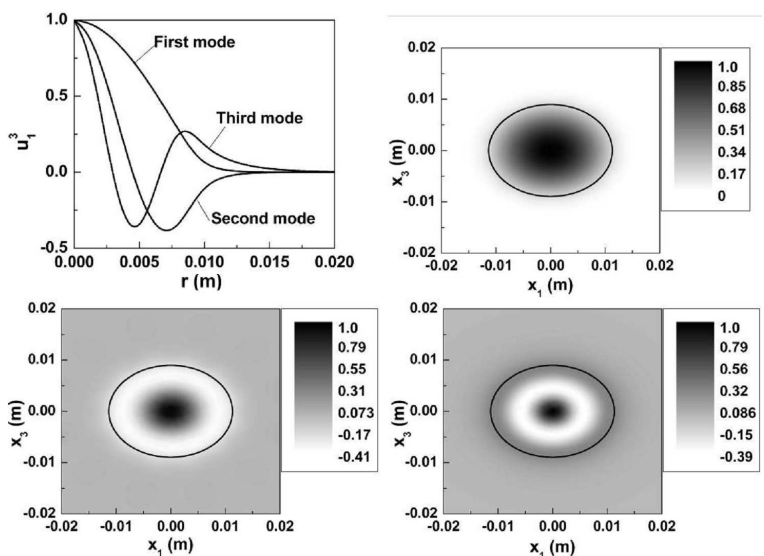

Fig. 4. Trapped modes when $n=3, R=0.01$, and $a=9 \mathrm{~mm}$.
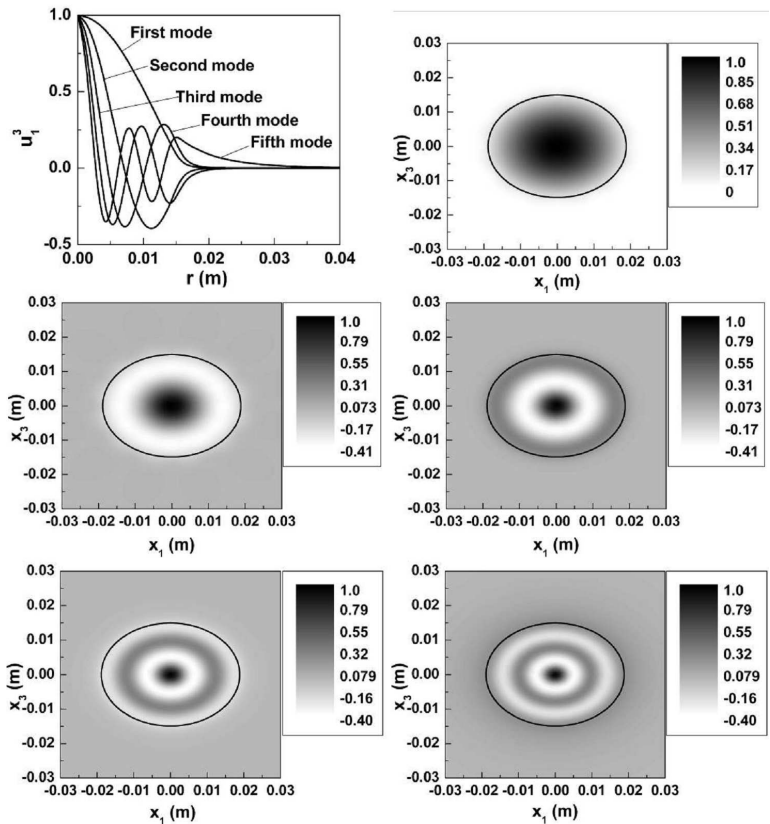

Fig. 5. Trapped modes when $n=3, R=0.01$, and $a=15 \mathrm{~mm}$. 
Still for the case of $n=1$, if we increase $a$ to $15 \mathrm{~mm}$, two trapped modes with $\omega=10321084.4041 / \mathrm{s}$ and $\omega=10407942.841 / \mathrm{s}$ are found. The two frequencies are close. The corresponding modes are shown in Fig. 3. The second mode has one nodal point in the radial direction.

Next we calculate overtone modes with $n>1$. When $R=0.01$ and $a=9 \mathrm{~mm}$, three trapped modes are found with frequencies $\omega=30986683.10$, 31086203.01, and $31253039.791 / \mathrm{s}$, respectively. These frequencies are slowly increasing and are about three times the frequencies of the fundamental modes. The corresponding modes are shown in Fig. 4. The first mode is trapped better than the corresponding one in Fig. 2.

If we increase $a$ to $15 \mathrm{~mm}$, five trapped modes are found with $\omega=30972311.01,31012083.41$, 31082852.72, 31182615.73 , and $\omega=31301843.251 / \mathrm{s}$, respectively. The corresponding modes are shown in Fig. 5.

\section{Conclusion}

Analytical solutions are obtained for trapped-energy resonators with elliptical electrodes. When the optimal electrodes are used, there is only one trapped mode which does not have nodal points under the electrodes. When electrodes larger than the optimal are used, there are more than one trapped modes and they have nodal points except the first mode. The frequencies of the fundamental modes with $n=1$ are close. The frequencies of the overtone modes with $n=3$ are about three times the frequencies of the fundamental.

\section{Acknowledgments}

This work was supported by the National Natural Science Foundation of China (No. 51404212).

\section{References}

[1] R.D. Mindlin, J. Acoust. Soc. Am. 43, 1329 (1968).

[2] K. Nakamura, R. Yasuike, K. Hirama, H. Shimizu, in: Proc. IEEE 44th Annual Symp. on Frequency Control, 1990, p. 372.

[3] Z.T. Yang, J.S. Yang, Y.T. Hu, Appl. Phys. Lett. 92, 103516 (2008).

[4] Z.T. Yang, J.S. Yang, IEEE Trans. Ultrason. Ferroelect. Freq. Contr. 56, 237 (2009).

[5] L.M. Xu, Y.L. Geng, Y.T. Hu, H. Fan, J.S. Yang, IEEE Trans. Ultrason. Ferroelect. Freq. Contr. 56, 875 (2009).

[6] T.F. Ma, C. Zhang, X.N. Jiang, G.P. Feng, Chin. Phys. B 20, 047701 (2011).

[7] W.J. Wang, R.X. Wu, J. Wang, J.K. Du, J.S. Yang, IEEE Trans. Ultrason. Ferroelect. Freq. Contr. 60, 1192 (2013).

[8] H.F. Tiersten, R.C. Smythe, J. Acoust. Soc. Am. 65, 1455 (1979). 